\title{
Genome-wide analysis in chicken reveals that local levels of genetic diversity are mainly governed by the rate of recombination
}

\author{
Carina F Mugal ${ }^{1}$, Benoit Nabholz ${ }^{1,2}$ and Hans Ellegren ${ }^{1 *}$
}

\begin{abstract}
Background: Polymorphism is key to the evolutionary potential of populations. Understanding which factors shape levels of genetic diversity within genomes forms a central question in evolutionary genomics and is of importance for the possibility to infer episodes of adaptive evolution from signs of reduced diversity. There is an on-going debate on the relative role of mutation and selection in governing diversity levels. This question is also related to the role of recombination because recombination is expected to indirectly affect polymorphism via the efficacy of selection. Moreover, recombination might itself be mutagenic and thereby assert a direct effect on diversity levels.

Results: We used whole-genome re-sequencing data from domestic chicken (broiler and layer breeds) and its wild ancestor (the red jungle fowl) to study the relationship between genetic diversity and several genomic parameters. We found that recombination rate had the largest effect on local levels of nucleotide diversity. The fact that divergence (a proxy for mutation rate) and recombination rate were negatively correlated argues against a mutagenic role of recombination. Furthermore, divergence had limited influence on polymorphism.

Conclusions: Overall, our results are consistent with a selection model, in which regions within a short distance from loci under selection show reduced polymorphism levels. This conclusion lends further support from the observations of strong correlations between intergenic levels of diversity and diversity at synonymous as well as non-synonymous sites. Our results also demonstrate differences between the two domestic breeds and red jungle fowl, where the domestic breeds show a stronger relationship between intergenic diversity levels and diversity at synonymous and non-synonymous sites. This finding, together with overall lower diversity levels in domesticates compared to red jungle fowl, seem attributable to artificial selection during domestication.
\end{abstract}

Keywords: Genetic diversity, Recombination rate, Chicken, Mutation, Selection, Gene density

\section{Background}

Modern population genetics have attempted to explain patterns of genetic variation in light of evolutionary forces thought to affect DNA sequence evolution. One obvious factor to form a candidate for governing local polymorphism levels is the rate of mutation since, in the absence of selection, sequence divergence should be proportional to the mutation rate [1]. Another obvious factor is selection since both positive and negative selection reduces levels of genetic diversity at target loci. Selection should also affect diversity levels in regions

\footnotetext{
* Correspondence: Hans. Ellegren@ebc.uu.se

'Department of Evolutionary Biology, Evolutionary Biology Centre, Uppsala University, Norbyvagen 18D, SE-752 36, Uppsala, Sweden

Full list of author information is available at the end of the article
}

linked to target loci. In the absence of recombination, the entire haplotype within which a selected allele is contained will be subject to change in frequency by selection. From this follows that recombination should itself be a factor of importance for levels of polymorphism. Specifically, when the local recombination rate is high, only regions within a relatively short physical distance from loci under selection are expected to show reduced polymorphism levels. There is well-developed theory for the expected effects of both types of selection relevant in this context, i.e. background selection arising from purifying selection [2] and selective sweeps arising from positive selection $[3,4]$.

Empirically, one of the clearest patterns that have emerged from studies of the distribution of levels of

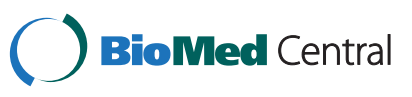


polymorphism across the genome is the positive effect of recombination rate on genetic diversity. This relationship was first observed in Drosophila melanogaster [5,6] and then confirmed in various organisms including mouse [7], human [8-10], nematodes of the genus Caenorabditis [11,12], sea beet [13] and grasses [14,15]. However, a direct effect of recombination on mutation rate, i.e., a neutral scenario, has also been proposed to explain the correlation between recombination and polymorphism $[9,16]$, although this possible mutagenic effect of recombination is debated [17-19]. A recent large-scale analysis failed to demonstrate a relationship between recombination hotspots and mutation rate in the human genome [20]. However, the fact that recombination rate as well as the rate of substitution often covary with several other genomic features impedes the understanding of any causal relationships $[9,21]$.

The possibility to capture patterns of sequence polymorphism across whole genomes allows critical tests of the importance of different evolutionary factors in shaping diversity levels [22]. This information is especially important when making inferences of selection, not least when it comes to detecting signs of positive selection in searches for candidate loci for adaptive evolution. It is then imperative that variation in polymorphism caused by different factors can be distinguished from each other. Here we analyze genome-wide patterns of genetic diversity in domestic chicken G. gallus domesticus and its wild ancestor the red jungle fowl G. gallus gallus. This system is of particular interest given that intense artificial selection during domestication may have left strong footprints on patterns of genetic diversity within the genome [23-26]. We examine how nucleotide diversity in chicken is related to recombination rate, divergence in intergenic regions and at synonymous and nonsynonymous sites, gene density and local GC content.

\section{Results}

Using published information on a total of 7.2 million chicken SNPs obtained from re-sequencing of pooled samples [25], we derived genome-wide estimates of local (1 Mb windows) diversity level for three chicken populations (red jungle fowl [RJF], broiler and layer) based on non-repetitive and putatively non-functional sites in the genome (see Materials and Methods). Basically, this represents diversity in intergenic regions, which in the following we simply refer to as 'diversity'. We also estimated diversity at synonymous and nonsynonymous sites, which we refer to as $\mathrm{p}_{\mathrm{S}}$ and $\mathrm{p}_{\mathrm{N}}$, respectively. Domestic breeds had lower diversity levels than RJF (electronic Additional file 1: Table S1), however, principal component analysis (PCA) showed that $88.8 \%$ of the variation was common to all three populations, represented by principal component (PC) I (see biplots in the Additional file 1: Figure S1). Pairwise correlations between local diversity level and $\mathrm{p}_{\mathrm{S}}$ and $\mathrm{p}_{\mathrm{N}}$, respectively, had correlation coefficients in the range 0.118-0.454 and generally showed a stronger relationship in the domestic breeds than in RJF. For all three populations the strongest correlation was found between diversity level and $\mathrm{p}_{\mathrm{S}}$ (Table 1).

In order to investigate the causes of local variation in diversity level we performed multi-linear regression analysis using a number of candidate explanatory variables selected to represent the effects of variation in mutation rate (intergenic divergence and the synonymous substitution rate, $\mathrm{d}_{\mathrm{S}}$ ), local DNA composition (GC content), recombination and selection (gene density and the nonsynonymous substitution rate, $\mathrm{d}_{\mathrm{N}}$ ), respectively. Here, intergenic divergence, $d_{S}$ and $d_{N}$ represent chickenspecific divergence estimates based on triple-alignments between chicken, turkey and zebra finch. Gene density represents the proportion of genic sites in the chicken sequence and GC content is computed based on nongenic sites in the chicken sequence. Then, using diversity levels as response variable, regression analysis was performed for each population separately as well as for PC I of diversity levels of all three populations. The latter was considered an appropriate representative of the genetic variation common to all populations. Recombination rate was found to be the main explanatory variable followed by gene density, both being positively correlated with diversity. GC content showed a statistically significant impact in RJF and for common genetic variation, whereas divergence showed a significant and unexpected negative impact in layer and for common genetic variation. $\mathrm{d}_{\mathrm{S}}$ and $\mathrm{d}_{\mathrm{N}}$ were not significantly correlated with diversity level at a $p$-value threshold of 0.001 in any population (Table 2). Overall, similar patterns were found for regression analysis based on $500 \mathrm{~kb}$ and $250 \mathrm{~kb}$ windows. However, the amount of genetic variation explained, represented by multiple $R^{2}$, decreased from

Table 1 Pairwise Pearson correlation coefficients (and associated $p$-values) between local diversity level and $p_{S}$ and $p_{N}$, based on $1 \mathrm{Mb}$ windows for three chicken populations

\begin{tabular}{lll}
\hline & Red jungle fowl & Broiler \\
\hline diversity level $-p_{S}$ & $0.237\left(1.17 \cdot 10^{-12}\right)$ & $0.454\left(<2.2 \cdot 10^{-16}\right)$ \\
diversity level $-p_{N}$ & $0.137\left(4.68 \cdot 10^{-05}\right)$ & $0.213\left(1.65 \cdot 10^{-10}\right)$ \\
$p_{S}-p_{N}$ & $0.118\left(4.36 \cdot 10^{-04}\right)$ & $0.403\left(<2.2 \cdot 10^{-16}\right)$ \\
\hline
\end{tabular}


Table 2 Estimates, by which we refer to multiple regression coefficients, and $p$-values in multi-linear regression analysis for six possible explanatory variables of chicken diversity levels in $1 \mathrm{Mb}$ windows

\begin{tabular}{|c|c|c|c|c|c|c|c|c|}
\hline & \multicolumn{2}{|c|}{ Red jungle fowl } & \multicolumn{2}{|c|}{ Broiler } & \multicolumn{2}{|c|}{ Layer } & \multicolumn{2}{|c|}{ Common } \\
\hline & Estimate & $p$-value & Estimate & $p$-value & Estimate & $p$-value & Estimate & $p$-value \\
\hline recombination rate & $3.29 \cdot 10^{-05}$ & $2.51 \cdot 10^{-16}$ & $3.07 \cdot 10^{-05}$ & $6.07 \cdot 10^{-16}$ & $2.32 \cdot 10^{-05}$ & $8.40 \cdot 10^{-10}$ & $5.03 \cdot 10^{-05}$ & $9.45 \cdot 10^{-16}$ \\
\hline divergence & $-7.94 \cdot 10^{-06}$ & $1.68 \cdot 10^{-02}$ & $-7.77 \cdot 10^{-06}$ & $1.33 \cdot 10^{-02}$ & $-1.82 \cdot 10^{-05}$ & $1.07 \cdot 10^{-08}$ & $-1.93 \cdot 10^{-05}$ & $2.09 \cdot 10^{-04}[27]$ \\
\hline gene density & $2.30 \cdot 10^{-05}$ & $4.45 \cdot 10^{-08}$ & $2.11 \cdot 10^{-05}$ & $1.07 \cdot 10^{-07}$ & $2.19 \cdot 10^{-05}$ & $3.95 \cdot 10^{-08}$ & $3.81 \cdot 10^{-05}$ & $6.54 \cdot 10^{-09}$ \\
\hline GC content & $-1.68 \cdot 10^{-05}$ & $7.10 \cdot 10^{-04}$ & $-1.50 \cdot 10^{-05}$ & $1.37 \cdot 10^{-03}$ & $-1.31 \cdot 10^{-05}$ & $5.49 \cdot 10^{-03}$ & $-2.60 \cdot 10^{-05}$ & $7.94 \cdot 10^{-04}$ \\
\hline$d_{s}$ & $-7.23 \cdot 10^{-06}$ & $8.18 \cdot 10^{-02}$ & $-7.18 \cdot 10^{-06}$ & $6.76 \cdot 10^{-02}$ & $-1.01 \cdot 10^{-05}$ & $1.03 \cdot 10^{-02}$ & $-1.41 \cdot 10^{-05}$ & $3.00 \cdot 10^{-02}$ \\
\hline \multirow[t]{3}{*}{$d_{N}$} & $-3.66 \cdot 10^{-06}$ & $3.70 \cdot 10^{-01}$ & $-4.64 \cdot 10^{-06}$ & $2.29 \cdot 10^{-01}$ & $-4.08 \cdot 10^{-06}$ & $2.92 \cdot 10^{-01}$ & $-7.13 \cdot 10^{-06}$ & $2.63 \cdot 10^{-01}$ \\
\hline & \multicolumn{2}{|c|}{ Multiple $R^{2}=0.1513$} & \multicolumn{2}{|c|}{ Multiple $R^{2}=0.1509$} & \multicolumn{2}{|c|}{ Multiple $R^{2}=0.1601$} & \multicolumn{2}{|c|}{ Multiple $R^{2}=0.1693$} \\
\hline & \multicolumn{2}{|c|}{$p<2.2 \cdot 10^{-16}$} & \multicolumn{2}{|c|}{$p<2.2 \cdot 10^{-16}$} & \multicolumn{2}{|c|}{$p<2.2 \cdot 10^{-16}$} & \multicolumn{2}{|c|}{$p<2.2 \cdot 10^{-16}$} \\
\hline
\end{tabular}

Common variation reflects PC I of diversity level of all three populations. Estimates and $p$-values significant at a threshold $<0.001$ are highlighted in bold.

$15 \%$ for analyses based on $1 \mathrm{Mb}$ windows to $8 \%$ and $5 \%$ for $500 \mathrm{~kb}$ and $250 \mathrm{~kb}$ windows, respectively. The decrease for the smaller window sizes is likely a result of a reduction in the signal-to-noise ratio, which motivated further analyses to be focused on $1 \mathrm{Mb}$ windows (for results based on $500 \mathrm{~kb}$ and $250 \mathrm{~kb}$ windows, see Additional file 1: Tables S2 and Additional file 1: Table S3).

As multi-linear regression analysis is sensitive to multicollinearity in the explanatory variables, we performed partial least square regression (PLSR) analysis, a regression setup that accounts for multi-collinearity in the explanatory variables and allows dissection of the interrelationships between explanatory variables. PLSR groups together explanatory variables into PCs based on their correlations with each other. Subsequent regression analysis and the number of significant PCs then illustrate the number of independent effects on the response variable. Each significant $\mathrm{PC}$ represents an independent effect by one of the contributors to the respective PC on the response variable, most likely the main contributor, which we refer to as the true explanatory variable. The remaining contributors to the $\mathrm{PC}$ are likely to be dragged by the true explanatory variable via their correlations to the true explanatory variable. As such PLSR enables us to quantify a lower bound of the amount of variation explained by the true explanatory variable, where the upper bound is given by the $R^{2}$ obtained by simple linear regression.

In agreement with the results of the multi-linear regression analysis, the PLSR analysis showed that recombination rate explained most of the variation in diversity level (6\%) (Table 3, and visualized in Figure 1 and Additional file 1: Figure S2). Gene density (3\%), GC content (3\%) and divergence (2\%) explained part of the variance, whereas the impact of $d_{S}$ and $d_{N}$ on diversity was $<1 \%$ and considered negligible. Figure 1 illustrates that most of the explanatory variables grouped together in PC I, which accentuates the complexity associated with determining independent effects of correlated explanatory variables. Based on PC I and in agreement with the multi-linear regression analysis the relationships between recombination rate, GC content and gene density, and diversity was positive, while divergence, $d_{S}$ and $d_{N}$ showed a negative relationship to diversity. Since recombination rate constitutes the main contributor to PC I it is likely to drag other correlated variables with it, like gene density, GC content and divergence. As recombination rate and gene density were positively correlated $(r=0.40, p<2.2 \mathrm{e}-16)$, this could explain why we find a positive correlation between gene density and diversity level, despite theory predicting the opposite. Similarly, this could lead to a negative correlation between divergence and diversity level, as recombination rate and divergence were negatively correlated $(r=-0.28, p<2.2 \mathrm{e}-16)$. Note that correlations between gene density and recombination rate, and between divergence and recombination rate, do not need to be causal. However, the negative correlation between recombination rate and divergence argues against a mutagenic effect of recombination (for point mutations) in birds. Also note that correlations might be caused

Table 3 Percentage of genetic variation explained by six possible explanatory variables according to PLSR analysis of chicken diversity level in $1 \mathrm{Mb}$ windows

\begin{tabular}{lcccc}
\hline & $\begin{array}{c}\text { Red jungle } \\
\text { fowl }\end{array}$ & Broiler & Layer & $\begin{array}{c}\text { Common } \\
\text { variation }\end{array}$ \\
\hline recombination rate & 6.30 & 6.16 & 4.48 & 6.34 \\
divergence & $(1.29)$ & $(1.37)$ & 3.62 & 2.06 \\
gene density & 3.02 & 2.91 & 2.93 & 3.31 \\
GC content & 3.12 & $(3.06)$ & $(3.13)$ & 3.48 \\
$\mathrm{~d}_{\mathrm{S}}$ & $(0.69)$ & $(0.65)$ & $(0.86)$ & $(0.78)$ \\
$\mathrm{d}_{\mathrm{N}}$ & $(0.49)$ & $(0.58)$ & $(0.66)$ & $(0.61)$ \\
\hline
\end{tabular}

Values in parentheses are for parameters that did not meet the $p<0.001$ threshold in multi-linear regression. 


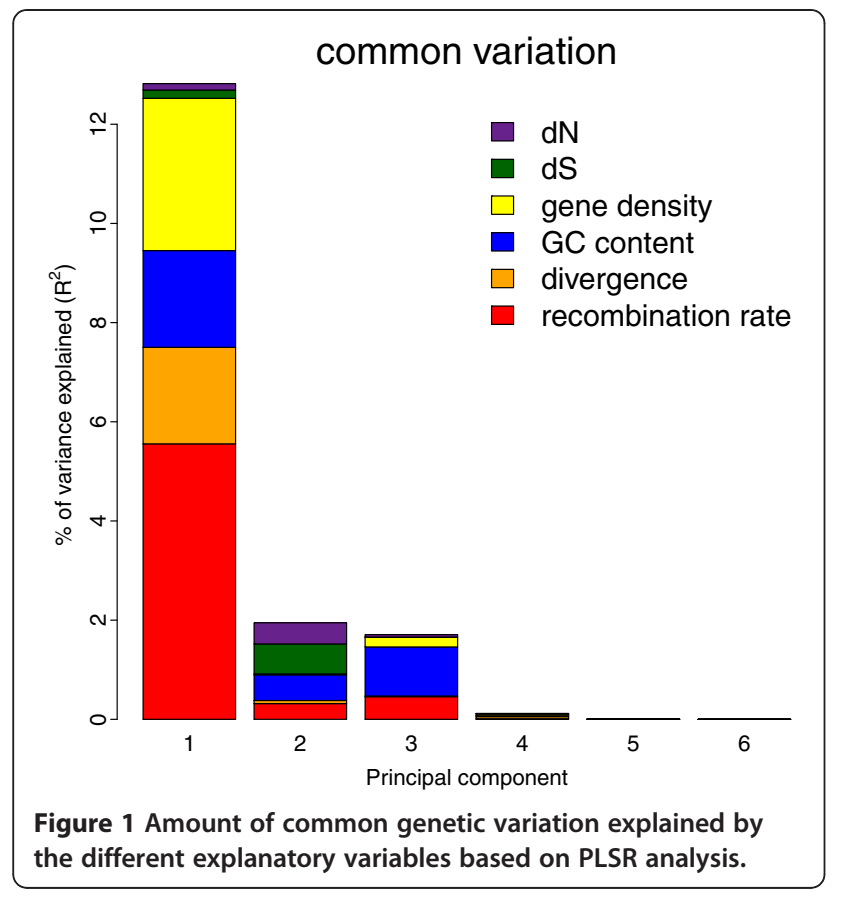

indirectly via a third parameter, like GC content (which itself is strongly correlated with recombination rate $[r=$ $0.61, p<2.2 \mathrm{e}-16]$, gene density $[r=0.66, p<2.2 \mathrm{e}-16]$ and divergence $[r=-0.27, p<2.2 \mathrm{e}-16])$, and thereby blur any true effects of mutation rate and/or gene density on diversity level. Based on PC II and III we again found a positive relationship between recombination rate and gene density, and diversity, and a negative relationship between divergence, $d_{S}$ and $d_{N}$, and diversity. However, opposite to the results based on PC I, GC content now showed a negative relationship to diversity. This suggests that after correction for the effect of recombination rate on diversity, GC content shows an independent negative effect on diversity.

We tested if the lack of, or a negative, correlation between divergence and diversity level could arise as a result of differences in the range of spatial variation of the two parameters by performing an autocorrelation analysis based on $100 \mathrm{~kb}$ windows. However, the patterns were similar for both diversity and divergence (Figure 2). Correlations decreased from $r>0.4$ between adjacent windows to $\mathrm{r}<0.1$ already at a distance of about $2 \mathrm{Mb}$, where $p$-values stayed low for a long distance.

Motivated by the results of the regression analysis, we investigated the impact of the explanatory variables for the possibility to identify candidate loci for selective sweeps. Based on the observation of locally reduced levels of genetic diversity, Rubin and colleagues identified 42 genomic regions as putative locations for selective sweeps in chicken [25]. Table 4 lists mean values for explanatory variables, and for $\mathrm{p}_{\mathrm{S}}$ and $\mathrm{p}_{\mathrm{N}}$, in these candidate regions together with genome-wide averages. This clearly highlights the importance of recombination rate for the ability to identify possible targets of positive selection: the mean recombination rate of candidate loci was reduced to $68 \%$ of the genome-wide average. Other explanatory variables also showed significant differences, but the effects were lower and are likely related to the correlation between explanatory variables.

\section{Discussion}

\section{Recombination as a determinant of levels of genetic diversity in chicken}

Diversity levels are expected to reflect the product of the mutation rate and $\mathrm{N}_{\mathrm{e}}$ [29]. As a consequence, intragenomic variation in the two latter parameters should lead to genomic heterogeneity in diversity levels. This heterogeneity can thus be framed either under a neutral scenario and reflect a mutation-driven pattern or under a model invoking natural selection causing variation in $\mathrm{N}_{\mathrm{e}}$, or both. It is well established that there is significant variation in the rate of mutation across the genome (e.g., [30]), providing a basis for variation in diversity level. $\mathrm{N}_{\mathrm{e}}$ is also expected to vary among genomic regions, in this case for reasons related to the incidence and efficiency of natural selection [29]. Importantly, with more functionally important sites follows more targets for selection. Moreover, when recombination rate is low, selection at linked sites will lower $\mathrm{N}_{\mathrm{e}}$ over larger physical distances along chromosomes [29]. Whether mutation or selection is the main factor governing diversity levels is a matter of on-going debate [22].

We found that recombination rate had the strongest effect on diversity levels in two domestic chicken breeds and in RJF. In contrast, divergence, taken as a proxy for the rate of mutation, had either no effect or an unexpected minor negative effect. This supports a selection model where the effect of background selection and/or selective sweeps is (physically) more widely reaching in genomic regions with low recombination rates. A positive correlation between diversity and recombination has been frequently observed in previous work, however, that the effect of recombination is indirect via selection has been difficult to disentangle from a possible direct mutagenic effect of recombination $[9,18,31]$. In our case, the negative correlation between recombination rate and divergence strengthens the selection model and argues against a mutagenic effect. Moreover, this interpretation is supported by the positive correlation of both $\mathrm{p}_{\mathrm{S}}$ and, in particular, $\mathrm{p}_{\mathrm{N}}$ with intergenic diversity level, showing that selective events that reduce the diversity within coding regions also reduce diversity at nearby linked sites (cf. [32]), or vice versa.

As mentioned above, selective effects are expected to increase with gene density, each coding site representing a 


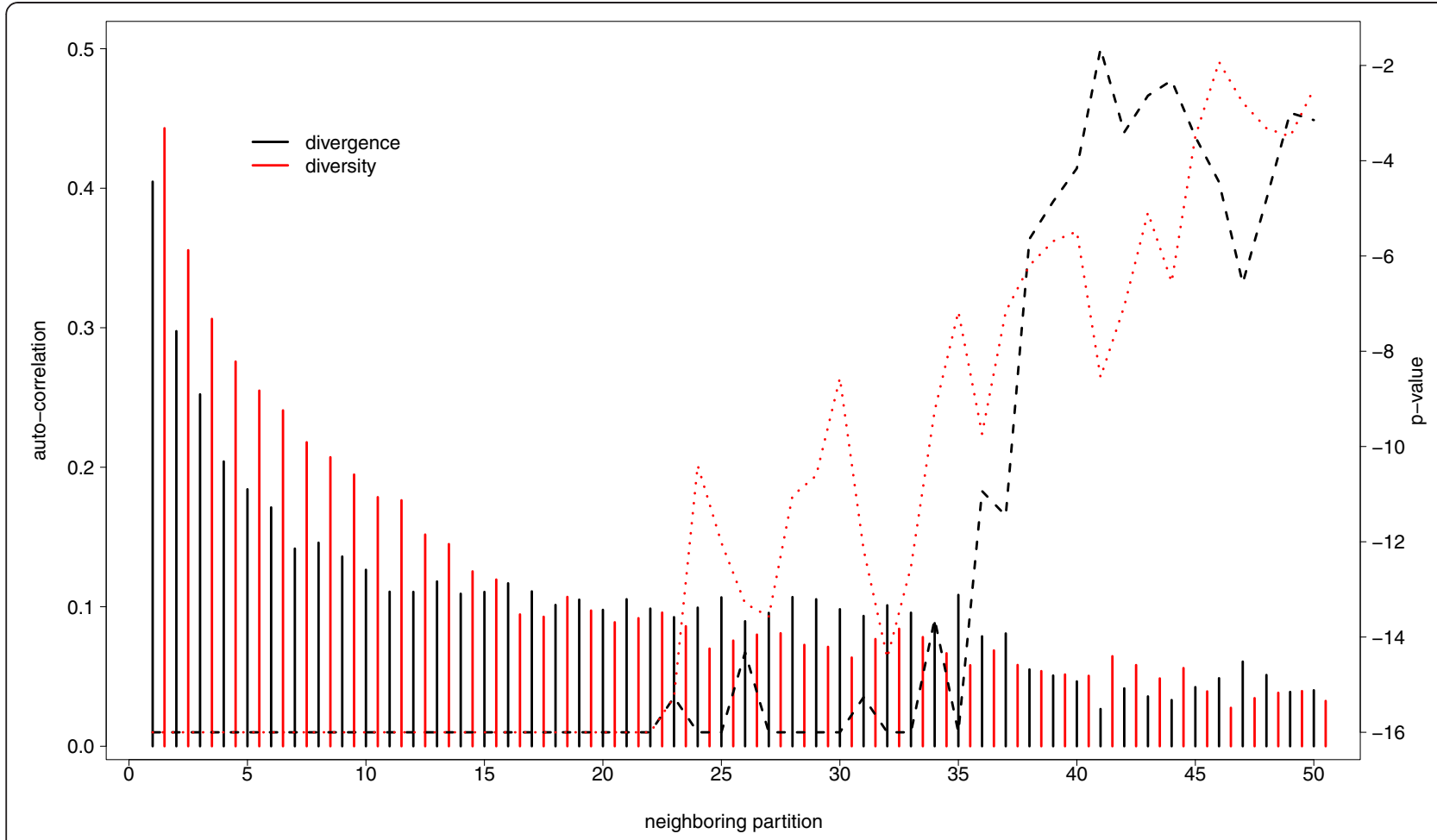

Figure 2 Pearson correlation coefficients (bars) and their $p$-values (dashed lines) for divergence (black) and diversity level (red) of neighboring windows of size $\mathbf{1 0 0} \mathbf{~ k b}$. The x-axis gives the number of the neighboring windows, starting from 1, i.e. nearest neighbor, up to 50 , i.e. windows separated by 49 windows. The $y$-axis indicates the correlation coefficient on the left side and the related $p$-value (log-scale) on the right.

potential target for selection. In this respect, the positive correlation between gene density and diversity goes against the theoretical expectation [33]. We suggest that this is a statistical artifact caused by collinearity of explanatory variables, like the relatively strong positive correlation between recombination rate and gene density $(r=0.4)$. Estimates

Table 4 Averages of diversity level, $p_{\mathrm{S}}, \mathrm{p}_{\mathrm{N}}$ and the six explanatory variables used for the regression analysis

\begin{tabular}{lccc}
\hline & Genome-wide & Candidate loci & $\boldsymbol{p}$ \\
\hline diversity level $\times 10^{4}$ & $3.69 / 3.42 / 3.06$ & $3.53 / 3.25 / 2.71$ & $<0.0001$ \\
$\mathrm{p}_{\mathrm{S}} \times 10^{4}$ & $5.12 / 5.11 / 4.66$ & $5.30 / 5.00 / 4.50$ & $0.338 / 0.395 / 0.167$ \\
$\mathrm{P}_{\mathrm{N}} \times 10^{5}$ & $5.50 / 5.38 / 5.01$ & $4.00 / 4.87 / 4.46$ & $0.005 / 0.006 / 0.006$ \\
recombination rate & 1.154 & 0.781 & $<0.0001$ \\
divergence & 0.038 & 0.040 & $<0.0001$ \\
gene density & 0.026 & 0.022 & $<0.0001$ \\
GC content & 0.408 & 0.392 & $<0.0001$ \\
$\mathrm{~d}_{\mathrm{S}} \times 10^{2}$ & 4.87 & 4.60 & $<0.0001$ \\
$\mathrm{~d}_{\mathrm{N}} \times 10^{2}$ & 0.90 & 0.77 & $<0.0001$ \\
\hline
\end{tabular}

The data are divided into genome-wide and candidate loci for selective sweeps identified by Rubin and colleagues [25]. $p$-values are based on bootstrapping. For diversity level, $\mathrm{p}_{\mathrm{S}}$ and $\mathrm{p}_{\mathrm{N}}$ means are reported for each of the three populations broiler, layer and RJF separately. Note that the absolute values of our diversity estimates are not directly comparable to previous estimates in chicken [28] since different sequencing methodologies and sampling design were used. from multiple regressions should be interpreted cautiously if explanatory variables are correlated since they may lead to spurious and non-causative correlations, which very well might be the case here. Moreover, this might explain a similarly surprising result recently reported for Asian rice (Oryza sativa) where the association between gene density and recombination rate could potentially explain a negative relationship between recombination rate and polymorphism [21], similar to earlier findings in Arabidopsis thaliana and $A$. lyrata $[34,35]$.

Several studies have shown that recombination rate is correlated with chromosome size [27,36,37], which is not unexpected given the requirement of at least one recombination event per chromosome (or chromosome arm) for successful meiosis; as a consequence, smaller chromosomes will have a higher recombination rate per physical distance compared to larger chromosomes. This is confirmed in our data, with a negative correlation between chromosome size and recombination rate $(r=-0.31, p<2.2 \mathrm{e}-16)$. As correlations are transitive relations, a correlation between chromosome size and recombination rate together with a correlation between recombination rate and diversity will lead to a correlation between chromosome size and diversity; this has been empirically demonstrated in previous 
analyses of birds [38]. In our main analysis we did not include chromosome size as candidate explanatory variable as we had no a priori reason to expect it to assert a direct effect on diversity level (in contrast to an indirect effect, via recombination). This was subsequently justified by a multi-linear regression analysis including chromosome size as explanatory variable (electronic Additional file 1: Table S4). This analysis suggested that in the RJF and broiler population there was no and in the layer population in fact an unexpected positive effect of chromosome size on diversity. Thus, we conclude that chromosome per se does not explain a negative correlation between chromosome size and diversity, as was also suggested by Megens et al. [39].

The absence of an effect of divergence on diversity levels We approximated local mutation rate by divergence estimates of the chicken branch after the split from turkey based on CpG-masked intergenic sequences as well as divergence at synonymous sites, $d_{S}$. Using these estimates we failed to find a persuasive effect of divergence on level of genetic diversity. This is surprising considering theory (that diversity and divergence are correlated is a basic tenet of the neutral theory of molecular evolution), and empirical evidence from some $[9,12,18,31]$ but not all previous studies $[6,40]$. The lack of a significant effect could be explained by several factors. First, in theory, it could reflect a lack of local variation in mutation rate across the chicken genome. However, this is clearly not in line with earlier observations from avian genomes $[41,42]$. Also, the auto-correlation analysis of divergence and diversity level (Figure 2) suggests that the two vary on a similar scale. Second, sequence features such as the local GC content appear to be strongly related to divergence in avian genomes. However, GC content is strongly correlated also with recombination rate via $\mathrm{GC}$-biased gene conversion (gBGC), a process linked to recombination mimicking natural selection and leading to high GC content in high recombining regions [43]. As a consequence, the covariation of recombination rate, GC content and divergence together with a strong impact of recombination rate on diversity could blur independent signals between divergence and diversity as suggested by the PLSR analysis (Figure 1). Thus, taken together the positive correlation between recombination rate and diversity and the absence of correlation between divergence and diversity support a selection model, where the weaker impact of mutation rate on diversity, if any, becomes indistinct by the stronger impact of recombination rate on diversity.

\section{The impact of genomic features on identifying targets of adaptive evolution}

There is considerable current interest in using population genomic data to identify regions that have been subject to recent events of positive selection. One means to do so is to search for outlier regions of nucleotide diversity, specifically regions of reduced diversity. The demonstration of recombination rate having a large impact on diversity level in the chicken genome should have at least two implications in this context. First, footprints of selection (selective sweeps) will be most easily seen in regions of low recombination, even if they occur less frequently in such regions due to Hill-Robertson interference. In addition, regions with low recombination and an associated reduced $\mathrm{N}_{\mathrm{e}}$ are more likely to show hard sweeps than soft sweeps [44]. This should be manifested both in diversity level being reduced over a larger genomic region and the reduction being visible over a longer time scale since the sweep. Second, and as consequence of the former, studies of the genomic distribution of adaptively evolving loci will be biased towards regions with low rate of recombination. This was confirmed when we analyzed the location of candidate loci for selective sweeps identified by Rubin et al. [25], with recombination rate being significantly lower in these candidate regions compared to the genomic average. This emphasizes the necessity of a rigorous statistical framework that incorporates genomic features such as recombination rate when interpreting polymorphism levels.

\section{The footprint of domestication on patterns of chicken diversity}

Although a significant part of the variation in diversity level was common to all three populations, we observed several important differences between domesticates and their wild ancestor. Rubin and colleagues found significantly lower overall heterozygosity in the two domestic breeds than in RJF [25]. Our results corroborate this observation, with the most pronounced difference seen in intergenic regions, with diversity level of the broiler and the layer being $83-93 \%$ of RJF. The difference is clearly in the expected direction given bottlenecks during domestication and genetic drift in closed commercial populations. Microsatellite-based genotyping has revealed this to be a common feature among chicken breeds [45] and, to a varying extent, the same trend has also been seen among other domestic animals and plants [46-48].

Strong artificial selection for traits of agronomical interest during domestication should also act to lower $\mathrm{N}_{\mathrm{e}}$ [25,49]. If artificial selection occurs frequently genome-wide it could create a stronger link between polymorphism at functional and neutral sites in domesticates than in natural populations [21]. In agreement with this prediction, the correlation of $\mathrm{p}_{\mathrm{S}}$ and $\mathrm{p}_{\mathrm{N}}$ to diversity level was stronger in the two domestic chicken breeds than in RJF (cf. Figure 2).

\section{Conclusions}

Two previous studies have sought to address the influence of recombination on chicken diversity levels. Fang 
et al. [50] used low-coverage genome sequence data from three birds to obtain polymorphism estimates and made pairwise linear regression between diversity and recombination rate estimates from a medium-density linkage map. Rao et al. [51] sequenced 15 introns, and used data from Sundström et al. [52] for another 14 introns, and performed pairwise linear regression between diversity and recombination rate, and between diversity and chicken-turkey divergence. Similar to our findings, these two studies reported a correlation between diversity and recombination. However, our study adds to previous work in several ways. Notably, by the combined use of a genome-wide approach for diversity estimation from population samples and with the access to divergence data from across the genome, we were able to address and quantify the role of mutation and recombination on diversity level in a rigorous statistical framework. In addition we considered possible impacts of $\mathrm{d}_{\mathrm{S}}, \mathrm{d}_{\mathrm{N}}$, gene density and the local GC content. Based on our analysis we suggest that local levels of genetic diversity in the chicken genome are mainly governed by the rate of recombination. The fact that divergence and recombination rate were negatively correlated argues against a mutagenic role of recombination and for a selection model. In support of the selection model, divergence, taken as a proxy for the rate of mutation, had either no effect or an unexpected minor negative effect. Moreover, by including genome-wide estimates of $\mathrm{p}_{\mathrm{S}}$ and $\mathrm{p}_{\mathrm{N}}$ we were able to directly study the role of selection and to integrate information from functional sites in the genome. In addition, the genome-wide approach allowed us to test for possible effects of various genomic features on the ability to identify target loci of adaptive evolution. Further, we showed that artificial selection during domestication is likely to explain several differences in levels of diversity between domestic breeds and the wild ancestor (red jungle fowl), for example a stronger relationship between recombination rate and intergenic diversity, as well as a stronger relationship between intergenic diversity levels and diversity at synonymous as well as nonsynonymous sites.

\section{Methods}

\section{Short read sequences and read mapping}

We used a dataset by Rubin and colleagues available at the European Nucleotide Archive (http://www.ebi.ac.uk/ ena/) under the study accession number SRP001870 [25]. This dataset is composed of 35 bp reads obtained by SOLiD sequencing technology of genomic DNA pools of unrelated chicken from the red jungle fowl (RJF; 8 males and 1 female) and two domesticated populations, broiler (24 males and 18 females) and layer (29 males) (accession numbers SRR035386, SRR035383 and SRR035384 for RJF, SRR035377, SRR035378, SRR035387, SRR035381,
SRR035382, SRR035379 and SRR035380 for broiler and SRR035375, SRR035376, SRR035389, SRR035390 and SRR035385 for layer).

The reads were mapped against the chicken reference genome (WUGSC 2.1, May 2006 version; [27]) downloaded at the UCSC Genome Browser website (http://genome.ucsc.edu/) [53]. The mapping was performed using the software BWA [46] allowing for a maximum of four mismatched bases and not allowing for insertions/deletions. Reads that mapped at several locations in the genome were excluded. Further, a genomic position had to fulfill four criteria in order to be included for downstream diversity level computation: (i) be covered by more than 4 and less than 50 reads; (ii) be outside of repeat sequences (based on the UCSC Genome Browser chicken repeat annotations); (iii) not correspond to a CpG prone site and (iv) be outside exons and untranslated regions (UTRs) that are likely to be affected by natural selection. In order to obtain data on synonymous and non-synonymous polymorphisms, we separately considered the corresponding positions within exons, still fulfilling criteria i) to iii).

Exons and UTRs coordinates were obtained through the BioMart query interface (http://www.ensembl.org/ biomart/martview) [54]. When no UTR was annotated for a transcript, we excluded $77 \mathrm{bp}$ upstream of the transcript (i.e. in $5^{\prime}$ direction) and 372 bp downstream of the transcript (i.e. in 3 ' direction), sizes corresponding to the mean lengths of annotated $5^{\prime}$ and $3^{\prime}$ UTRs in chicken, respectively. A $\mathrm{CpG}$ prone site was defined as any $\mathrm{C}$ followed by a $G$ or any $G$ preceded by a $C$, as well as any $\mathrm{C} / \mathrm{T}$ polymorphism followed by a $\mathrm{G}$ or any $\mathrm{G} / \mathrm{A}$ polymorphism preceded by a $\mathrm{C}$, following [55].

\section{SNP calling and estimates of diversity level}

To be called a SNP, we followed the approach by Rubin et al. [25], that is we applied the criterion that the alternative nucleotide state, i.e. the non-reference allele, must be supported by at least three reads different to the nucleotide state found in the reference genome. While diversity estimates were obtained for RJF, broiler and layer populations separately, the support of the alternative nucleotide state was based on combined data of multiple populations. For example, consider a hypothetical position with a $\mathrm{C}$ in the reference genome. If the broiler population had two reads with $\mathrm{A}$ and one read with $\mathrm{C}$ at this position, and the layer population had one with $\mathrm{A}$ and two with $\mathrm{C}$, the position was called a SNP in both layer and broiler populations, because the number of non-reference alleles (i.e., A) summed up to 3 . Once the SNPs were called, we validated our SNP calls with those SNPs called by Rubin et al. [25], and only used consistently called SNPs.

After the validation step, we computed the number of SNPs per non-overlapping window (SNP density) for 
$1 \mathrm{Mb}, 500 \mathrm{~kb}, 250 \mathrm{~kb}$ and $100 \mathrm{~kb}$ windows. Additionally, we computed the mean coverage per window as the average read depth per validated genomic position. These tasks were performed using in-house perl scripts. We determined the number of synonymous and nonsynonymous SNPs per synonymous and non-synonymous sites by in-house $\mathrm{C}++$ scripts incorporating the $\mathrm{Bio}++$ library [56].

In order to correct SNP density estimates for variation in coverage, we estimated diversity level following an approach by Cutter and Moses [32]:

$$
\hat{\theta}=[S N P] / \log (n-1)
$$

where $[S N P]$ represents the SNP density and $n$ denotes the mean coverage. This computation was performed for all four window sizes for intergenic SNPs, where in the following $\theta$ is referred to as diversity level. For synonymous and non-synonymous polymorphisms the estimation was only performed for $1 \mathrm{Mb}$ windows in order to ensure a reasonable signal-to-noise ratio. Here, the mean coverage was exclusively based on the read depth of synonymous and non-synonymous sites, respectively. Synonymous and non-synonymous diversity levels are in the following referred to as $\mathrm{p}_{\mathrm{S}}$ and $\mathrm{p}_{\mathrm{N}}$, respectively. Note that the absolute values of our diversity estimates are not directly comparable to other studies in chicken, because we used NGS data based on pooled samples rather than Sanger sequencing data and we employed stringent filtering criteria.

\section{Sequence data}

Sequence alignments of orthologous intergenic regions for chicken, turkey (Meleagris gallopavo) and zebra finch (Taeniopygia guttata) were retrieved from whole-genome alignments from the Ensembl database release 61 via the Ensembl perl Application Programme Interfaces (APIs). We partitioned the whole-genome alignments into the four window sizes stated above, respectively, each with reference to the chicken genome. Then positions of transcribed regions including UTRs were established and masked with reference to the chicken genome. For each dataset, we restricted the data to windows with a minimum of 10,000 unambiguous sites, of which there were 1,038 windows of size $1 \mathrm{Mb}, 2,040$ of size $500 \mathrm{~kb}, 3,986$ of size $250 \mathrm{~kb}$ and 9,205 of size $100 \mathrm{~kb}$.

Coding sequence (CDS) alignments of orthologous genes in chicken, turkey and zebra finch were retrieved through the protein trees from the Ensembl database release 61 via Ensembl perl APIs. Orthologous genes were restricted to one-to-one orthologs, as defined in the Ensembl database. Alignments of one-to-one orthologs were then concatenated based on the windows defined for intergenic regions; for genes spanning more than one window, the different parts were assigned to the respective window. Windows containing no one-to-one orthologs were discarded for downstream analysis. Sequence alignments were cleaned for possible misaligned sites running Gblocks with default parameter settings [57].

\section{Estimation of divergence, $d_{N}, d_{s}$, gene density and recombination rate}

We estimated chicken-specific divergence for intergenic regions as the branch length between chicken and its common ancestor with turkey after all sites showing a CpG in any of chicken, turkey and zebra finch had been masked from the 3-way alignments. Estimation of branch length was based on the PAML software package version 4.1 and the general time-reversible substitution model implemented in baseml. CpG sites were masked from the alignments in order to avoid substitution rate variation caused by hypermutability of $\mathrm{CpG}$ sites and thus divergence being affected by the local CpG content.

We estimated chicken-specific rates of non-synonymous $\left(d_{\mathrm{N}}\right)$ and synonymous $\left(\mathrm{d}_{\mathrm{S}}\right)$ substitution for the concatenated CDS alignments using PAML software package version 4.1. CDS alignments were concatenated in a given window of size $1 \mathrm{Mb}, 500 \mathrm{~kb}$ and $250 \mathrm{~kb}$, respectively. To estimate chicken-specific $d_{S}$ and $d_{N}$, we then used the branch-model implemented in codeml allowing the $\mathrm{d}_{\mathrm{N}} / \mathrm{d}_{\mathrm{S}}$ ratio to vary between the chicken branch and the remaining tree.

We estimated gene density as the proportion of exonic sites within a particular window. We also included UTRs and exon-intron boundaries as "genic" sites, as they might represent functionally important sequences. For the exon-intron boundaries, we included $10 \mathrm{bp}$ of intronic sequence after the end and before the start of each exon [58].

We computed the sex-averaged chicken recombination rate using data from Groenen et al. [36] and the WUGSC 2.1 chicken assembly. Recombination rate per $1 \mathrm{Mb}$ window was computed as the mean recombination rate (genetic distance/physical distance) between markers weighted by the physical distance between markers, ranging from $0-28.6 \mathrm{cM}$ per $1 \mathrm{Mb}$ window (a histogram of recombination rate is provided in the Additional file 1: Figure S3).

\section{Statistical analysis}

To investigate the degree of common variation in genetic diversity between the three chicken populations we performed PCA of local diversity level for $1 \mathrm{Mb}, 500 \mathrm{~kb}$ and $250 \mathrm{~kb}$ non-overlapping windows. The computation of the PCs was performed via an eigenvalue-decomposition of the associated covariance matrix as implemented in the "princomp" function of the statistical software package $\mathrm{R}$ version 2.9.2. The degree of common variation was then 
defined as the leading PC, i.e. PC I, and as a local measure of the common genetic variation in the three populations we projected diversity levels on PC I, which can be seen as a smoothing function through genetic diversity in all three chicken populations.

We performed multi-linear regression analysis for diversity level grouped into four groups: common genetic variation in diversity level (PC I) and variation in each of the three chicken populations separately. For all four groups we conducted regression analysis based on 880 out of 1,038 non-overlapping windows of size $1 \mathrm{Mb}$, where data on the six possible explanatory variables recombination rate, divergence, gene density, GC content, $\mathrm{d}_{\mathrm{S}}$ and $\mathrm{d}_{\mathrm{N}}$ were available. We further conducted regression analysis for 1,623 windows of size $500 \mathrm{~kb}$ and 2,651 windows of size $250 \mathrm{~kb}$. We transformed the explanatory variables in order to reduce the skewness in their distributions. Recombination rate was log-transformed to base 10, after adding a constant of 1 in order to allow for zero rate values. All the other explanatory variables were transformed by the square root. Regression analysis was then performed after Z-transformation of the explanatory variables, which means standardization of the mean value to 0 and of the standard deviation to 1 .

We performed PLSR analysis, a regression setup that accounts for multi-collinearity in the explanatory variables [59]. As stated above for the multi-linear regression analysis, explanatory variables were first transformed to reduce the skewness in their distributions and then Ztransformed. In addition, also diversity level estimates were also Z-transformed. PLSR was then conducted for diversity level estimates based on $1 \mathrm{Mb}$ windows for each of the four groups of genetic variation separately.

We performed an autocorrelation analysis of local diversity level and divergence based on $100 \mathrm{~kb}$ windows. This was done computing Pearson correlation coefficients and their $p$-values for measurements of nearest neighboring windows $(\mathrm{k}=1)$ up to windows lying $5 \mathrm{Mb}$ apart $(\mathrm{k}=50)$.

Genomic regions of candidate selective sweeps identified by Rubin and colleagues were mapped onto the $1 \mathrm{Mb}$ windows used throughout our analysis [25]. Averages of diversity levels, $\mathrm{p}_{\mathrm{S}}$ and $\mathrm{p}_{\mathrm{N}}$, as well as averages of the six explanatory variables used in the above described regression analysis were determined for the candidate loci as the arithmetic means of the respective $1 \mathrm{Mb}$ windows. Genome-wide averages of the same variables were determined as the arithmetic means over all windows. To assess the significance in the difference between the averages for the candidate loci and the genome-wide averages we bootstrapped the genome-wide averages based on a sample size of 9999 and computed $p$-values based on their bootstrap confidence intervals.

All statistical analyses were performed with the software package $\mathrm{R}$ version 2.9.2.

\section{Additional file}

\begin{abstract}
Additional file 1: Figure S1. Biplots of the first two principal components (PCs) for diversity level across the genome for three window sizes, $1 \mathrm{Mb}, 500 \mathrm{~kb}$ and $250 \mathrm{~kb}$. In each graph one black dot represents one window, where observations of diversity level in the three populations are projected into the space of the first two PCs. The red arrows labeled RJF, Broiler and Layer display the loadings for diversity level in the respective population. Arrows which represent the loadings of the three populations on PC I and PC II showed a strong component along $P C I$ all pointing in the same direction and a much weaker along PC II. Figure S2. Amount of variation in local diversity level in each of the three chicken populations explained by the different explanatory variables based on PLSR analysis. Figure S3. Histogram of recombination rate in CM per $1 \mathrm{Mb}$ window. Table S1: Summary of genome-wide averages and 95\% bootstrap confidence intervals (Cls) of diversity level, $p_{S}, p_{N}$ and $p_{N} / p_{S}$ estimates for the three different chicken populations. For diversity level data for three different window sizes are included whereas for $p_{S}, p_{N}$ and $p_{N} / p_{S}$ only $1 \mathrm{Mb}$ windows are considered due smaller sample sizes. Table S2: Estimates and p-values in a multi-linear regression analysis for six possible explanatory variables of chicken diversity level in $500 \mathrm{~kb}$ windows for the three populations and for common genetic variation. Estimates and $p$-values significant at a threshold $<0.001$ are highlighted in bold. Table S3: Estimates and p-values in a multi-linear regression analysis for six possible explanatory variables of chicken diversity level in $250 \mathrm{~kb}$ windows for the three populations and for common genetic variation. Estimates and $p$-values significant at a threshold $<0.001$ are highlighted in bold. Table S4: Estimates and $p$-values in multi-linear regression analysis for seven possible explanatory variables of chicken diversity levels in $1 \mathrm{Mb}$ windows. Common variation reflects $\mathrm{PC}$ I of diversity level of all three populations. Estimates and $p$-values significant at a threshold $<0.001$ are highlighted in bold.
\end{abstract}

\section{Abbreviations}

RJF: Red jungle fowl; PCA: Principal component analysis; PC: Principal component; PLSR: Partial least square regression; $\mathrm{N}_{\mathrm{e}}$ : Effective population size; $d_{S}$ : Synonymous divergence; $d_{N}$ : Non-synonymous divergence; $\mathrm{p}_{\mathrm{s}}$ : Synonymous diversity; $\mathrm{P}_{\mathrm{N}}$ : Non-synonymous diversity; gBGC: GC-biased gene conversion; API: Application Programme Interface; CDS: Coding sequence.

\section{Competing interests}

The authors declare that they have no competing interests.

\section{Authors' contributions}

$\mathrm{BN}$ and CFM performed the bioinformatics analysis. CFM performed the statistical analysis. CFM and HE conceived and designed the study. BN, CFM and HE drafted the manuscript and all authors approved the final manuscript.

\section{Acknowledgements}

We thank Yves Clément for helpful discussions on the statistical analysis. Financial support was obtained from the European Research Council, the Knut and Alice Wallenberg Foundation and the Swedish Research Council.

\section{Author details}

${ }^{1}$ Department of Evolutionary Biology, Evolutionary Biology Centre, Uppsala University, Norbyvagen 18D, SE-752 36, Uppsala, Sweden. ${ }^{2}$ Present address: Institut des Sciences de l'Evolution, Université Montpellier II, Place Eugène Bataillon, 34095, Montpellier cedex 5, France.

Received: 11 June 2012 Accepted: 4 February 2013 Published: 8 February 2013

\section{References}

1. Kimura M: Evolutionary rate at the molecular level. Nature 1968, 217(5129):624-626.

2. Charlesworth B, Morgan MT, Charlesworth D: The effect of deleterious mutations on neutral molecular variation. Genetics 1993, 134(4):1289-1303. 
3. Smith JM, Haigh J: The hitch-hiking effect of a favourable gene. Genet Res 1974, 23(1):23-35.

4. Andolfatto P: Adaptive hitchhiking effects on genome variability. Curr Opin Genet Dev 2001, 11(6):635-641.

5. Berry AJ, Ajioka JW, Kreitman M: Lack of polymorphism on the drosophila fourth chromosome resulting from selection. Genetics 1991, 129(4):1111-1117.

6. Begun DJ, Aquadro CF: Levels of naturally occurring DNA polymorphism correlate with recombination rates in D. Melanogaster. Nature 1992 356(6369):519-520.

7. Nachman MW: Patterns of DNA variability at X-linked loci in Mus domesticus. Genetics 1997, 147(3):1303-1316.

8. Nachman MW, Bauer VL, Crowell SL, Aquadro CF: DNA variability and recombination rates at X-Linked loci in humans. Genetics 1998, 150(3): 1133-1141.

9. Hellmann I, Prafer K, Ji H, Zody MC, Pääbo S, Ptak SE: Why do human diversity levels vary at a megabase scale? Genome Res 2005, 15(9):1222-1231.

10. Spencer CCA, Deloukas P, Hunt S, Mullikin J, Myers S, Silverman B, Donnelly P, Bentley $D, M c V e a n ~ G:$ The influence of recombination on human genetic diversity. PLoS Genetics 2006, 2(9):e148.

11. Cutter $A D$, Payseur BA: Selection at linked sites in the partial selfer caenorhabditis elegans. Mol Biol Evol 2003, 20(5):665-673.

12. Cutter AD, Choi JY: Natural selection shapes nucleotide polymorphism across the genome of the nematode caenorhabditis briggsae. Genome Res 2010, 20(8):1103-1111.

13. Kraft T, Säll T, Magnusson-Rading I, Nilsson N-O, Halldén C: Positive correlation between recombination rates and levels of genetic variation in natural populations of sea beet (Beta vulgaris subsp. maritima). Genetics 1998, 150(3):1239-1244.

14. Dvorák J, Luo M-C, Yang Z-L: Restriction fragment length polymorphism and divergence in the genomic regions of high and low recombination in self-fertilizing and cross-fertilizing Aegilops species. Genetics 1998, 148(1):423-434

15. Tenaillon MI, Sawkins MC, Long AD, Gaut RL, Doebley JF, Gaut BS: Patterns of DNA sequence polymorphism along chromosome 1 of maize (Zea mays ssp. Mays L.). Proc Natl Acad Sci U S A 2001, 98(16):9161-9166.

16. Lercher MJ, Hurst LD: Human SNP variability and mutation rate are higher in regions of high recombination. Trends in Genetics 2002, 18(7):337-340.

17. Begun DJ, Holloway AK, Stevens K, Hillier LW, Poh Y-P, Hahn MW, Nista PM, Jones CD, Kern AD, Dewey CN, et al: Population genomics: whole-genome analysis of polymorphism and divergence in drosophila simulans. PLOS Biology 2007, 5(11):e310.

18. Kulathinal RJ, Bennett SM, Fitzpatrick CL, Noor MAF: Fine-scale mapping of recombination rate in drosophila refines its correlation to diversity and divergence. Proc Natl Acad Sci U S A 2008, 105(29):10051-10056.

19. Huang S-W, Friedman $\mathrm{R}, \mathrm{Yu}$ N, Yu A, Li W-H: How strong is the mutagenicity of recombination in mammals? Mol Biol Evol 2005, 22(3): 426-431.

20. 1000 GC: A map of human genome variation from population-scale sequencing. Nature 2010, 467(7319):1061-1073.

21. Flowers JM, Molina J, Rubinstein S, Huang P, Schaal BA, Purugganan MD: Natural selection in gene-dense regions shapes the genomic pattern of polymorphism in wild and domesticated rice. Mol Biol Evol 2012, 29(2): 675-687.

22. Gossmann TI, Woolfit M, Eyre-Walker A: Quantifying the variation in the effective population size within a genome. Genetics 2011, 189(4):1389-1402.

23. Vigouroux $Y$, McMullen M, Hittinger $C T$, Houchins $K$, Schulz L, Kresovich $S$, Matsuoka $Y$, Doebley J: Identifying genes of agronomic importance in maize by screening microsatellites for evidence of selection during domestication. Proc Natl Acad Sci U S A 2002, 99(15):9650-9655.

24. Clark RM, Linton E, Messing J, Doebley JF: Pattern of diversity in the genomic region near the maize domestication gene tb1. Proc Natl Acad Sci U S A 2004, 101(3):700-707.

25. Rubin CJ, Zody MC, Eriksson J, Meadows JR, Sherwood E, Webster MT, Jiang $\mathrm{L}$, Ingman M, Sharpe $\mathrm{T}$, Ka S, et al: Whole-genome resequencing reveals loci under selection during chicken domestication. Nature 2010 464(7288):587-591.

26. Innan $H$, Kim Y: Pattern of polymorphism after strong artificial selection in a domestication event. Proc Natl Acad Sci U S A 2004, 101(29):10667-10672

27. ICGSC: Sequence and comparative analysis of the chicken genome provide unique perspectives on vertebrate evolution. Nature 2004, 432(7018):695-716.
28. ICPMC: A genetic variation map for chicken with 2.8 million singlenucleotide polymorphisms. Nature 2004, 432(7018):717-722.

29. Betancourt AJ, Welch JJ, Charlesworth B: Reduced effectiveness of selection caused by a lack of recombination. Curr Biol 2009, 19(8):655-660

30. Hodgkinson A, Eyre-Walker A: Variation in the mutation rate across mammalian genomes. Nat Rev Genet 2011, 12(11):756-766.

31. Slotte T, Bataillon T, Hansen TT, St. Onge K, Wright SI, Schierup MH: Genomic determinants of protein evolution and polymorphism in Arabidopsis. Genome Biol Evol 2011, 3:1210-1219.

32. Cutter AD, Moses AM: Polymorphism, divergence, and the role of recombination in saccharomyces cerevisiae genome evolution. Mol Biol Evol 2011, 28(5):1745-1754

33. Payseur BA, Nachman MW: Gene density and human nucleotide polymorphism. Mol Biol Evol 2002, 19(3):336-340.

34. Nordborg M, Hu T, Ishino Y, Jhaveri J, Toomajian C, Zheng HG, Bakker $E$, Calabrese P, Gladstone J, Goyal R, et al: The pattern of polymorphism in arabidopsis thaliana. PLOS Biology 2005, 3(7):1289-1299.

35. Kawabe A, Forrest A, Wright SI, Charlesworth D: High DNA sequence diversity in pericentromeric genes of the plant arabidopsis lyrata. Genetics 2008, 179(2):985-995

36. Groenen MAM, Wahlberg P, Foglio M, Cheng HH, Megens H-J, Crooijmans RPMA, Besnier F, Lathrop M, Muir WM, Wong GK-S, et al: A high-density SNPbased linkage map of the chicken genome reveals sequence features correlated with recombination rate. Genome Res 2009, 19(3):510-519.

37. Groenen MAM, Cheng HH, Bumstead N, Benkel BF, Briles WE, Burke T, Burt DW Crittenden LB, Dodgson J, Hillel J, et al: A consensus linkage map of the chicken genome. Genome Res 2000, 10(1):137-147.

38. Huynh LY, Maney DL, Thomas JW: Contrasting population genetic patterns within the white-throated sparrow genome (zonotrichia albicollis). BMC Genet 2010, 11:96.

39. Megens HJ, Crooijmans RPMA, Bastiaansen JWM, Kerstens HHD, Coster A Jalving $\mathrm{R}$, Vereijken A, Silva $\mathrm{P}$, Muir WM, Cheng HH, et al: Comparison of linkage disequilibrium and haplotype diversity on macro- and microchromosomes in chicken. BMC Genet 2009, 10.

40. Roselius K, Stephan W, Städler T: The relationship of nucleotide polymorphism, recombination rate and selection in wild tomato species. Genetics 2005, 171(2):753-763.

41. Axelsson E, Webster MT, Smith NGC, Burt DW, Ellegren $\mathrm{H}$ : Comparison of the chicken and turkey genomes reveals a higher rate of nucleotide divergence on microchromosomes than macrochromosomes. Genome Res 2005, 15(1):120-125.

42. Webster MT, Axelsson E, Ellegren $\mathrm{H}$ : Strong regional biases in nucleotide substitution in the chicken genome. Mol Biol Evol 2006, 23(6):1203-1216.

43. Duret L, Galtier N: Biased gene conversion and the evolution of mammalian genomic landscapes. Annual Rev Genomics Human Genetics 2009, 10(1):285-311.

44. Hermisson J, Pennings PS: Soft sweeps: molecular population genetics of adaptation from standing genetic variation. Genetics 2005, 169(4):2335-2352.

45. Granevitze Z, Hillel J, Chen GH, Cuc NTK, Feldman M, Eding H, Weigend S: Genetic diversity within chicken populations from different continents and management histories. Anim Genet 2007, 38(6):576-583.

46. vonHoldt BM, Pollinger JP, Lohmueller KE, Han E, Parker HG, Quignon P, Degenhardt JD, Boyko AR, Earl DA, Auton A, et al: Genome-wide SNP and haplotype analyses reveal a rich history underlying dog domestication. Nature 2010, 464(7290):898-902.

47. Amaral AJ, Ferretti L, Megens H-J, Crooijmans RPMA, Nie H, Ramos-Onsins SE, Perez-Enciso M, Schook LB, Groenen MAM: Genome-wide footprints of pig domestication and selection revealed through massive parallel sequencing of pooled DNA. PLoS One 2011, 6(4):e14782

48. Haudry A, Cenci A, Ravel C, Bataillon T, Brunel D, Poncet C, Hochu I, Poirier S, Santoni S, Glémin S, et al: Grinding up wheat: A massive loss of nucleotide diversity since domestication. Mol Biol Evol 2007, 24(7):1506-1517.

49. Wright SI, Bi IV, Schroeder SG, Yamasaki M, Doebley JF, McMullen MD, Gaut BS: The effects of artificial selection on the maize genome. Science 2005, 308(5726):1310-1314

50. Fang L, Ye J, Li N, Zhang Y, Li S, Wong G, Wang J: Positive correlation between recombination rate and nucleotide diversity is shown under domestication selection in the chicken genome. Chin Sci Bull 2008, 53(5): 746-750.

51. Rao $Y$, Sun $L$, Nie $Q$, Zhang $X$ : The influence of recombination on SNP diversity in chickens. Hereditas 2011, 148(2):63-69. 
52. Sundström H, Webster MT, Ellegren H: Reduced variation on the chicken Z chromosome. Genetics 2004, 167:377-385.

53. Fujita PA, Rhead B, Zweig AS, Hinrichs AS, Karolchik D, Cline MS, Goldman M, Barber GP, Clawson H, Coelho A, et al: The UCSC genome browser database: update 2011. Nucleic Acids Res 2011, 39(suppl 1):D876-D882.

54. Smedley D, Haider S, Ballester B, Holland R, London D, Thorisson G, Kasprzyk A: BioMart - biological queries made easy. BMC Genomics 2009, 10(1):22.

55. Keightley PD, Gaffney DJ: Functional constraints and frequency of deleterious mutations in noncoding DNA of rodents. Proc Natl Acad Sci U S A 2003, 100(23):13402-13406.

56. Dutheil J, Gaillard S, Bazin E, Glemin S, Ranwez V, Galtier N, Belkhir K: Bio++: a set of $\mathrm{C}++$ libraries for sequence analysis, phylogenetics, molecular evolution and population genetics. BMC Bioinforma 2006, 7(1):188.

57. Castresana J: Selection of conserved blocks from multiple alignments for their use in phylogenetic analysis. Mol Biol Evol 2000, 17(4):540-552

58. Abril JF, Castelo R, Guigó R: Comparison of splice sites in mammals and chicken. Genome Res 2005, 15(1):111-119.

59. Naes T, Martens H: Multivariate calibration .2. Chemometric methods. Trac-Trend Anal Chem 1984, 3(10):266-271.

doi:10.1186/1471-2164-14-86

Cite this article as: Mugal et al:: Genome-wide analysis in chicken reveals that local levels of genetic diversity are mainly governed by the rate of recombination. BMC Genomics 2013 14:86.

\section{Submit your next manuscript to BioMed Central and take full advantage of:}

- Convenient online submission

- Thorough peer review

- No space constraints or color figure charges

- Immediate publication on acceptance

- Inclusion in PubMed, CAS, Scopus and Google Scholar

- Research which is freely available for redistribution 